EPJ Web of Conferences 19, 10006 (2012)

DOI: $10.1051 /$ epjconf/20121910006

(C) Owned by the authors, published by EDP Sciences, 2012

\title{
Applying the manifold theory to Milky Way models: First steps on morphology and kinematics
}

\author{
M. Romero-Gómez ${ }^{1, a}$, E. Athanassoula ${ }^{2}$, T. Antoja ${ }^{3}$ and F. Figueras ${ }^{1}$ \\ ${ }^{1}$ Dept. d'Astronomia i Meteorologia \& Institut de Ciències del Cosmos, \\ Universitat de Barcelona, Martí i Franquès 1, 08028 Barcelona, Spain \\ ${ }^{2}$ Laboratoire d'Astrophysique de Marseille, UMR6110, CNRS/Université de Provence, \\ 38 rue Frédéric Joliot Curie, 13388 Marseille, France \\ ${ }^{3}$ Kapteyn Astronomical Institute, University of Groningen, PO Box 800, 9700 AV Groningen, \\ The Netherlands
}

\begin{abstract}
We present recent results obtained by applying invariant manifold techniques to analytical models of the Milky Way. It has been shown that invariant manifolds can reproduce successfully the spiral arms and rings in external barred galaxies. Here, for the first time, we apply this theory to Milky Way models. We select five different models from the literature and, using the parameters chosen by the authors of the papers, and three different cases, namely Case 1, where only the COBE/DIRBE bar is included in the potential; Case 2, when the COBE/DIRBE and the Long bar are aligned, and Case 3, when the COBE/DIRBE bar and the Long bar are misaligned. We compute in each case and for each model the orbits trapped by the manifolds. In general, the global morphology of the manifolds can account for the 3-kpc arms and for the Galactic Molecular Ring.
\end{abstract}

\section{INTRODUCTION}

We study the effects of the COBE/DIRBE bar and/or the Long bar in the inner 2-6 kpc of the Milky Way. We apply the manifold theory to five analytic Galactic models that consist of an axisymmetric plus a bar-like component and three Cases. In Case 1 we include only the COBE/DIRBE bar and in Cases 2 and 3 we include also the Long bar. In Case 2 we follow the general morphology of external galaxies $[1,2]$ and we align the two bars [2-4], while in Case 3 we place them $30^{\circ}$ degrees apart, as claimed by observations [5, 6]. The main characteristics of all models and cases are studied in detail in [4] and the values for one of the models described there (PMM04 [7]) are given in Table 1.

\section{MORPHOLOGY AND KINEMATICS GENERATED BY THE MANIFOLDS}

For each of the models, we compute the orbits trapped by the invariant manifolds associated to periodic orbits of the unstable Lagrangian points $L_{1}$ and $L_{2}$. These orbits are shown for the model PMM04 and Case 2 in Fig. 1. It is given in the frame that rotates with the bar. Here the bar lies at $20^{\circ}$ from the Sun - Galactic Centre line. Note that the main morphology is that of an inner ring elongated along the COBE/DIRBE and Long bar with a maximum axis of $\sim 4 \mathrm{kpc}$ and an outer ring with a major axis almost perpendicular to that of the inner ring, with a length of $6.5 \mathrm{kpc}$ and an axis ratio of 0.75 . In the middle and right panels of Fig. 1, we plot the (Galactic longitude, line-of-sight velocity) diagram (hereafter (1,v)

\footnotetext{
ae-mail: mromero@am.ub.es
}

This is an Open Access article distributed under the terms of the Creative Commons Attribution-Noncommercial License 3.0, which permits unrestricted use, distribution, and reproduction in any noncommercial medium, provided the original work is properly cited. 
Table 1. Our model and its main characteristics. $R_{0}$ is the Solar radius, $\Omega_{b}$ is the pattern speed of the bar (note that in Cases 2 and 3, both bars rotate at the same pattern speed). $a$ and $a_{L}$ denote the semi-major axis of the COBE/DIRBE and Long bar in kpc, respectively, while, $M_{b}$ and $M_{b_{L}}$ give the mass of the COBE/DIRBE and Long bar in $M_{\odot}$, respectively.

\begin{tabular}{llllllll}
\hline Model & Bar & $R_{0}$ & $\Omega_{b}$ & $a$ & $a_{L}$ & $M_{b}$ & $M_{b_{L}}$ \\
\hline PMM04 & Composite & 8.5 & 60. & 3.1 & 4.5 & $10^{10}$ & $6 \times 10^{9}$ \\
\hline
\end{tabular}
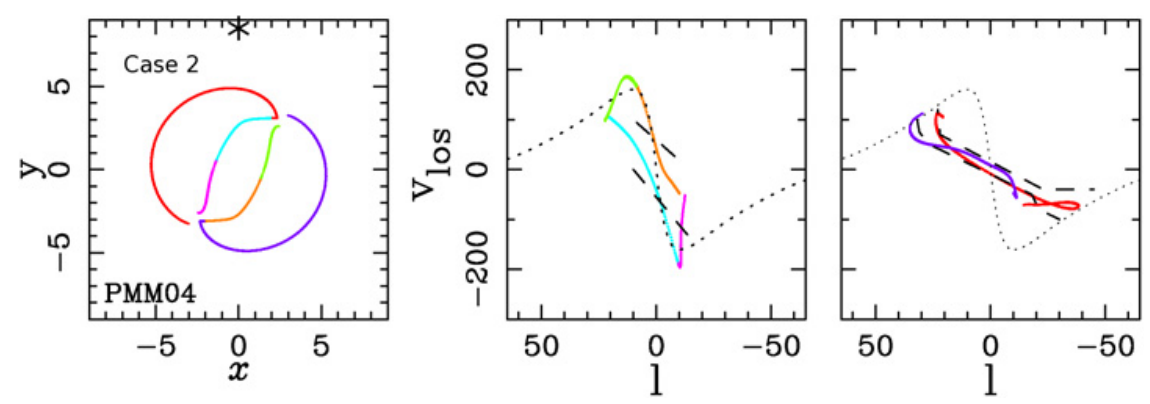

Figure 1. Morphology and kinematics in Case 2 and PMM04 model. Left panel: orbits in the (x,y) plane, the colours showing different parts of the rings. The asterisk marks the position of the Sun. Middle and right panels: $(1, v)$ diagram of the inner and outer manifolds, respectively. The circular terminal velocity is given by the black dotted line.

diagram) of the inner and outer rings, respectively. The dashed lines in these panels sketch the observed position of the 3-kpc near and far arm and of the Galactic Molecular Ring, respectively [8, 9], and the dotted lines show the circular terminal velocity.

\section{CONCLUSIONS}

After the study performed in [4] and summarised here, we can conclude that the manifolds are able to reproduce the observed kinematics of the gas of the inner $2-6 \mathrm{kpc}$ of the Milky Way Galactic disc. The outer ring reproduces well the overdensity in the (1,v)-diagram corresponding to the Galactic Molecular Ring. In some cases, the size and elongation of the inner ring reproduces the near and far 3-kpc arm. The study also reveals that models with less strong bars cannot reproduce the line-of-sight velocities at $l=0^{\circ}$, suggesting that the strength of the model Galactic bars is mild. The introduction of the Long bar has an effect on the elongation of the inner orbits and increases the line-of-sight velocities. The value of the angular separation between the bars introduces changes both in the morphology and in the kinematics.

\section{References}

[1] E. Athanassoula, MNRAS, 358, (2005) 1477

[2] I. Martínez-Valpuesta \& O. Gerhard, ApJL 734, (2011) L20

[3] E. Athanassoula, (2006) arXiv:astro-ph/0610113

[4] M. Romero-Gomez, E. Athanassoula, T. Antoja, F. Figueras, MNRAS, (2011) in press, arXiv:1108.0660

[5] P.L. Hammersley, F. Garzón, T.J. Mahoney, M. López-Corredoira, M.A.P. Torres, MNRAS 317, (2000) L45

[6] R. Benjamin et al., ApJ 630, (2005) L149

[7] B. Pichardo, M. Martos, E. Moreno, ApJ 609, (2004) 144 (PMM04)

[8] T.M. Dame, D. Hartmann, P. Thaddeus, ApJ 547, (2001) 792

[9] T.M. Dame \& D. Thaddeus, ApJ 683, (2008) 143 